\section{Origen, componentes y posibles mecanismos de acción de la dieta mediterránea}

\author{
INÉS URQUIAGA ${ }^{1, \mathrm{a}}$, GUADALUPE ECHEVERRÍA ${ }^{1, \mathrm{~b}}$, \\ CATALINA DUSSAILLANT ${ }^{1}$, ATTILIO RIGOTTI ${ }^{1,2}$
}

\section{Origin, components and mechanisms of action of the Mediterranean diet}

The Mediterranean diet is currently considered a healthy dietary pattern. It includes a great variety of foods, which are eaten in moderation and within a positive social environment. The generic term "Mediterranean diet" was born after the "Seven Countries Study" led by Ancel Keys around 1960. This dietary pattern is characterized by a high intake of fruits and vegetables, whole grains, legumes, nuts, fish, white meats and olive oil. It also includes moderate consumption of fermented dairy products, low intake of red meat and drinking wine with moderation during meals. Nutritionally, this diet is low in saturated fats and animal protein, high in antioxidants, fiber and monounsaturated fats, and exhibits an adequate omega-6/omega-3 fatty acid balance. The main bioactive compounds, which explain the health benefits of this dietary pattern, are antioxidants, fiber, monounsaturated and omega-3 fatty acids, phytosterols and probiotics. This diet is not exclusively confined to the Mediterranean Basin. Central Chile has a Mediterranean climate and our agriculture and culinary traditions are similar to those found in Mediterranean countries. Therefore, it is fundamental to increase awareness about the richness of our natural produce as well as our culinary culture, which may bring many health benefits and improve the quality of life in our population.

(Rev Med Chile 2017; 145: 85-95)

Key words: Diet, Mediterranean; Diet Therapy; Food; Quality of Life.
${ }^{1}$ Centro de Nutrición Molecular y Enfermedades Crónicas, Escuela de Medicina, Pontificia Universidad Católica de Chile. Santiago, Chile.

${ }^{2}$ Departamento de Nutrición, Diabetes y Metabolismo, Escuela de Medicina, Pontificia Universidad Católica de Chile. Santiago, Chile.

aBioquímica, PhD en Ciencias Biológicas.

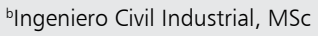

Recibido el 9 de noviembre de 2015, aceptado el 22 de junio de 2016.

Correspondencia a: Dr. Attilio Rigotti Departamento de Nutrición, Diabetes y Metabolismo, Escuela de Medicina, Pontificia Universidad Católica de Chile. Marcoleta 367, interior, 2do Piso, Santiago Código Postal 8330024, Chile. Teléfono + 56223543832 arigotti@med.puc.cl
I

as enfermedades crónicas son actualmente la principal causa de morbimortalidad en Chile y el mundo, constituyendo una preocupación creciente en salud pública. De hecho, la enfermedad cardiovascular (ECV), la diabetes mellitus tipo 2 y el cáncer podrían prevenirse mejorando el estilo de vida de la población, lo que incluye el consumo de una dieta saludable ${ }^{1}$.

Una alimentación de mala calidad es un determinante modificable de diferentes patologías crónicas. Así, se ha visto que dietas ricas en grasas saturadas, ácidos grasos poliinsaturados omega-6, ácidos grasos trans e hidratos de carbono refina- dos, junto con un bajo aporte de antioxidantes y fibra, son perjudiciales para la salud ${ }^{2}$.

Por el contrario, el consumo de una alimentación saludable como la dieta mediterránea (DMed) ha mostrado numerosos beneficios para la salud. Entre varios efectos favorables, esta dieta reduce la prevalencia de síndrome metabólico, diabetes mellitus, ECV, cáncer mamario y deterioro psicoorgánico ${ }^{3-8}$. En este artículo revisaremos el origen del concepto de DMed, sus componentes esenciales y los posibles mecanismos que explican su efecto beneficioso en salud. Además, discutiremos la aplicabilidad del consumo de este patrón 
alimentario en nuestro país. Esta revisión acompaña a otro artículo donde se revisa la evidencia científica actual que respalda los beneficios de la DMed en la prevención y manejo de las enfermedades crónicas?.

\section{Origen y concepto de la dieta mediterránea}

El concepto de DMed se popularizó a mediados del siglo pasado como consecuencia del "Estudio de los Siete Países", liderado por Ancel Keys entre 1958 y 1964, que comparó los hábitos dietéticos de diferentes cohortes de Estados Unidos de Norteamérica (EE. UU.), Japón, Finlandia, Holanda, la antigua Yugoslavia, Italia y Grecia ${ }^{10}$. El seguimiento de estas poblaciones durante 5-15 años confirmó una menor mortalidad por enfermedad coronaria y general y una mayor expectativa de vida en los países mediterráneos, particularmente en Grecia ${ }^{11}$.

El análisis global del "Estudio de los Siete Países" permitió definir los componentes de la dieta que se asociaban con una mayor expectativa de vida y una menor incidencia de enfermedades crónicas. De esta forma, se llegó a la conclusión que este patrón dietario, asociado especialmente a beneficios cardiovasculares, se caracterizaba por una baja ingesta de carnes rojas y un alto consumo de frutas, verduras, legumbres, aceite de oliva, frutos secos y pescado. Las características saludables de esta dieta de los habitantes de la costa europea del mar Mediterráneo llevaron a Keys a denominar a esta forma de alimentación como "dieta mediterránea".

Posteriormente, se estableció que no existe una DMed única, ya que los alimentos que forman parte de este patrón muestran variaciones entre los países de la cuenca mediterránea. Sin embargo, la dieta de los países europeos como España, Francia, Italia y Grecia, también norte de Âfrica y Medio Oriente, presenta características comunes que incluyen: 1) baja ingesta de grasas saturadas procedentes de mantequilla, leche entera y carnes rojas; 2) alto consumo de grasas monoinsaturadas, contenidas principalmente en aceite de oliva; 3 ) balance adecuado de ácidos grasos poliinsaturados (omega-6 versus omega-3), principalmente por el consumo de pescados, mariscos y frutos secos; 4) bajo aporte de proteína derivada de animales terrestres, especialmente carnes rojas; 5) alta inges- ta de antioxidantes, presentes en frutas, verduras, vino, aceite de oliva virgen, especias y hierbas, $y$ 6) alto consumo de fibra, proveniente de alimentos de origen vegetal como verduras, frutas, cereales integrales, legumbres y frutos secos ${ }^{12}$.

Esta definición de DMed no solamente incluye una gran variedad de alimentos, sino que también recomienda una determinada frecuencia de consumo de éstos (Tabla 1). Adicionalmente, la DMed pone énfasis en la preparación de comidas sabrosas, más que en la restricción estricta de alimentos específicos. Así, esta dieta propone un consumo diverso de alimentos, con moderación y acompañados de vino tinto, disfrutando socialmente en un ambiente tranquilo y relajado, todo lo cual, junto a la práctica de actividad física regular, forma parte del estilo de vida mediterráneo. En 2010, esta dieta fue declarada Patrimonio Cultural Inmaterial de la Humanidad.

\section{Componentes bioactivos y mecanismos de acción de la dieta mediterránea}

Los componentes bioactivos mejor reconocidos como responsables de los efectos beneficiosos de esta dieta son los antioxidantes, la fibra y los fitoesteroles, provenientes de productos vegetales, verduras, frutas, legumbres, aceite de oliva virgen y vino; los ácidos grasos monoinsaturados presentes en el aceite de oliva; los ácidos grasos omega-3 provenientes de productos marinos y de frutos secos; y los probióticos derivados de alimentos fermentados como queso y yogur, entre otros (Tabla 2) ${ }^{13}$. A través de múltiples mecanismos sinérgicos derivados de la presencia de estos componentes, la DMed genera diversos cambios fisiológicos y metabólicos (p.e., mejoría en perfil de antioxidantes, perfil lipídico, presión arterial, inflamación y coagulación, además de modulación en la expresión génica), que, en último término, determinan un menor riesgo de enfermedades crónicas, mayor longevidad y mejor calidad de vida. Es así como estudios de intervención con DMed han mostrado mejorías significativas en función endotelial, sensibilidad a insulina y presión arterial, con una reducción significativa de las concentraciones séricas de proteína $\mathrm{C}$ reactiva de alta sensibilidad (hs-CRP), IL-6, IL-7, IL-18, VCAM-1y ICAM-1 y un incremento en adiponectina ${ }^{14-18}$. Asimismo, se ha observado una mejor 
Tabla 1. Componentes alimentarios y frecuencia de consumo característicos de las dietas mediterráneas

\begin{tabular}{|ll|}
\hline Grupo de alimentos & Frecuencia de consumo \\
\hline Verduras & Diaria, en abundante cantidad, 3 o más porciones al día, crudas y cocidas \\
\hline Frutas & Diaria, en abundante cantidad, 2 o más porciones al día \\
\hline Aceite de oliva & Diaria, 3 a 6 cucharadas al día, como principal fuente de grasa \\
\hline Cereales, principalmente pan y pastas & Diaria, 3 a 4 veces al día en moderada cantidad \\
\hline Legumbres & Al menos 3 veces por semana \\
\hline Frutos secos & Al menos 3 veces por semana \\
\hline $\begin{array}{l}\text { Productos lácteos, principalmente } \\
\text { fermentados, yogurt y quesos }\end{array}$ & Diaria, 2 a 4 porciones al día \\
\hline Huevos & 1 a 4 unidades por semana \\
\hline Pescados y mariscos & 2 a 4 veces por semana \\
\hline Aves & 2 a 4 veces por semana \\
\hline Carnes rojas y procesadas & 1 vez por semana o menos \\
\hline Vino & Diaria, consumo moderado ( 1 copa al día para mujeres y 2 copas al día para \\
\hline homecias y condimentos & hombres) y en forma regular, principalmente con las comidas \\
\hline
\end{tabular}

Tabla 2. Componentes bioactivos aportados por alimentos de la dieta mediterránea

\begin{tabular}{|ll|}
\hline Componente bioactivo de la DMed & Alimentos que lo contienen \\
\hline Antioxidantes & $\begin{array}{l}\text { Verduras, frutas, aceite de oliva virgen, frutos secos, legumbres, especias } \\
\text { y condimentos, vino }\end{array}$ \\
\hline Fibra & Verduras, frutas, cereales integrales, legumbres \\
\hline Fitoesteroles & Cereales, nueces y frutos secos, legumbres y aceites vegetales \\
\hline Ácidos grasos monoinsaturados & Aceite de oliva \\
\hline $\begin{array}{l}\text { Ácidos grasos omega } 3 \\
\text { Probióticos }\end{array}$ & $\begin{array}{l}\text { Pescados y mariscos, y frutos secos } \\
\text { Lácteos fermentados derivados de la leche, como yogurt y quesos, olivas o } \\
\text { aceitunas, alcaparras, vinagre y vino }\end{array}$ \\
\hline
\end{tabular}

relación entre colesterol-HDL (c-HDL) y colesterol total, junto con un aumento en el tamaño de las partículas de HDL y un desplazamiento de las subfracciones de colesterol LDL (c-LDL) hacia un perfil menos aterogénico ${ }^{19,20}$ con menores niveles de LDL oxidadas ${ }^{21-24}$.

Característicamente, la DMed recomienda un bajo consumo de alimentos reconocidamente deletéreos para la salud, como grasas saturadas e hidratos de carbono simples, los cuales han sido asociados consistentemente con mayor riesgo de ateroesclerosis y diabetes mellitus, respectivamen- te. Asimismo, el menor consumo de sal como aderezo en las comidas, la cual es reemplazada por especias y condimentos, determina un menor consumo total de sodio. Esto, junto a una mayor ingesta de potasio y magnesio, proveniente de frutas y verduras, beneficia al sistema cardiovascular disminuyendo la presión arterial y preservando la función endotelial.

La forma particular de preparar la comida en la cocina mediterránea tiene efectos positivos para la salud. El consumo de salsa de tomate o sofrito -aceite de oliva, cebolla, ajo y tomate-, base de 
los guisos mediterráneos, se asocia con menor riesgo cardiovascular ${ }^{25,26}$. La evidencia muestra que el proceso de cocción libera licopeno y otros compuestos, aumentando su biodisponibilidad. Igualmente, se ha reportado que el consumo de alimentos ricos en licopeno reduce el riesgo de cáncer, especialmente cáncer de próstata ${ }^{27-32}$. Precisamente, en el estudio PREDIMED (Prevención con Dieta Mediterránea), el cuestionario validado para evaluar el cumplimiento de la dieta mediterránea adjudica puntaje a los que consumen sofrito dos o más veces por semana ${ }^{33,34}$.

\section{Antioxidantes}

La DMed aporta una elevada cantidad de antioxidantes de origen vegetal, entre ellos, vitamina $\mathrm{C}$, vitamina $\mathrm{E}, \beta$-caroteno, glutatión, licopeno y polifenoles (fenoles ácidos y flavonoides), que contribuyen a reducir el daño oxidativo a nivel celular y sistémico y, por ende, a disminuir la incidencia de enfermedades crónicas ${ }^{35-38}$. El estudio PREDIMED demostró que los grupos intervenidos que evidenciaron una mayor ingesta de polifenoles totales presentaron una reducción de $46 \%$ en el riesgo de $\mathrm{ECV}$, al compararse con el quintil de menor ingesta de polifenoles ${ }^{39}$.

Los polifenoles pueden tener un efecto antioxidante, así como participar en cascadas de señalización intracelular, mediante su interacción con receptores celulares o proteínas, modulando de esta manera la expresión génica y determinando respuestas fisiológicas beneficiosas ${ }^{40,41}$. De hecho, se han reportado interacciones flavonoide-proteína en más de 40 sistemas enzimáticos en mamíferos ${ }^{42,43}$. Algunas de las enzimas inhibidas por los polifenoles, con resultados beneficiosos en salud cardiovascular, son la 5-lipoxigenasa, la xantina oxidasa, la NADPH oxidasa y la enzima convertidora de angiotensina I (ECA) $)^{44,45}$.

Las dos principales vías de señalización que son moduladas por algunos flavonoides son la vía del factor de transcripción NF-kB, en que se inhibe su unión al ADN y su actividad transcripcional, y la vía de la sirtuina (SIRT), en que se incrementa su actividad histona deacetilasa dependiente de NAD y se regula la transcripción de múltiples proteínas $^{46,47}$.

Los efectos protectores de los polifenoles sobre la ECV han sido atribuidos a la mejoría que producen en la función endotelial, a la disminución de la presión arterial y a la mejoría del perfil lipídico ${ }^{48}$. De esta forma, la ingesta de bebidas ricas en polifenoles produce vasodilatación arterial en humanos ${ }^{49,50}$. Adicionalmente, estudios in vitro muestran un aumento en la actividad y expresión de la óxido nítrico sintasa endotelial (eNOS) e inhibición de la NADPH oxidasa endotelial en respuesta a polifenoles, lo que podría explicar, en parte, su efecto modulador sobre la función endotelial ${ }^{50,51}$. Por otro lado, los efectos anti-hipertensivos descritos son mediados por la supresión de la síntesis de endotelina-1, y la inhibición de la enzima convertidora de angiotensina-1 (ECA) ${ }^{52}$.

En el tracto gastrointestinal superior, los polifenoles, al igual que otros antioxidantes, actuarían reduciendo los hidroperóxidos de los alimentos ingeridos y formando complejos con metales de transición, contribuyendo a contrarrestar el estrés oxidativo postprandial ${ }^{53}$. Precisamente, el consumo de vino tinto con las comidas, un componente tradicional de la DMed, evita la oxidación de ácidos grasos poliinsaturados dietarios, reduciendo la aparición postprandial de lipoperóxidos, malondialdehido (MDA) y LDL oxidadas en el plasma $^{54-57}$. Adicionalmente, los polifenoles son parcialmente metabolizados por la flora microbiana colónica ${ }^{58}$, generando mediadores bioactivos benéficos para la salud humana.

\section{Fibra}

La DMed considera el consumo de alimentos ricos en fibra dietética. La fibra alimentaria corresponde a la fracción comestible de las plantas que resiste la digestión y absorción en el intestino delgado humano y que experimenta una fermentación parcial o total en el intestino grueso. Algunas fibras son insolubles, como la celulosa, y provienen principalmente de cereales integrales. Otras fibras son solubles, como la inulina, y son obtenidas con el consumo de verduras, frutas, legumbres, cebada y avena.

Los estudios prospectivos muestran que la ingesta de fibra dietética se asocia inversamente con el riesgo de eventos coronarios, tanto fatales como no fatales ${ }^{59-61}$, estimándose una disminución del riesgo cardiovascular de $9 \%$ por cada $7 \mathrm{~g} /$ día de ingesta de fibra total ${ }^{59}$. Asimismo, los estudios de intervención muestran efectos beneficiosos de la fibra dietética sobre los factores de riesgo cardiovascular, como las dislipidemias, la hipertensión arterial y la disglicemia. Esto se debe a que la fibra 
disminuye la absorción de grasas y azúcares de los alimentos, lo que contribuye a regular los niveles de colesterol y de glucosa plasmáticos, previniendo así la ECV y la diabetes.

La fibra alimentaria también facilita el tránsito intestinal y previene el estreñimiento y el cáncer digestivo. Adicionalmente, es un factor determinante de saciedad, disminuyendo la ingesta energética y facilitando el control del peso corporal ${ }^{62,63}$.

\section{Fitoesteroles}

Los esteroles vegetales son componentes importantes de una dieta basada en vegetales, siendo particularmente abundante en cereales, frutos secos, legumbres y aceites vegetales. Los fitoesteroles remedan la estructura del colesterol, compitiendo con este lípido por su incorporación en las micelas de sales biliares, disminuyendo su absorción intestinal y los niveles séricos de colesterol.

En estudios poblacionales, la ingesta de fitoesteroles dietarios se relaciona inversamente con los niveles plasmáticos de colesterol ${ }^{64}$. Además, la intervención con DMed suplementada con aceite de oliva o frutos secos del estudio PREDIMED demostró una mayor ingesta de fitoesteroles (76 y $158 \mathrm{mg} /$ día, respectivamente) versus el grupo control (15 mg/día), y una asociación inversa significativa entre ingesta de fitoesteroles y niveles de colesterol LDL plasmático ${ }^{65}$.

\section{Ácidos grasos monoinsaturados}

El efecto protector del aceite de oliva virgen, un componente clave de la DMed, sobre la ECV, algunos tipos de cáncer y enfermedades cognitivas relacionadas con la edad, se debería a su composición grasa rica en ácidos grasos monoinsaturados, especialmente ácido oleico (70\%), baja en ácidos grasos saturados $(5 \%)$ y poliinsaturados $(10-15 \%)$ y alta en compuestos polifenólicos antioxidantes. El ácido oleico tiene efectos beneficiosos sobre el perfil de lípidos plasmáticos, disminuyendo el colesterol total, el c-LDL y los triglicéridos e incrementando el c-HDL. Los compuestos polifenólicos antioxidantes, como el hidroxitirosol y la oleouropeina, entre otros, protegen de la oxidación a las partículas de LDL y tienen un efecto antitrombótico, antiinflamatorio, anticancerígeno, vasodilatador e hipotensor ${ }^{66}$. Además, el consumo de aceite de oliva virgen aumenta la sensibilidad periférica a la acción de la insulina y modula la inflamación y el estrés oxidativo ${ }^{66-68}$.

\section{Ácidos grasos omega-3}

El desarrollo de la actividad agrícola y la agroindustria facilita el consumo de dietas ricas en ácidos grasos saturados y desequilibrada en ácidos grasos omega- 6 versus omega- 3 , con un mayor aporte de los primeros con respecto de los segundos. Así, el uso de granos en alimentación del ganado y aves determina la producción de carnes, leche y huevos con alto contenido de ácidos grasos omega- 6 . Como consecuencia, la dieta actual de EE. UU. tiene una razón de ácidos grasos omega-6/omega-3 entre 15-16:1 versus 9-10:1 en Chile y 1-4:1 en Grecia y Japón ${ }^{69}$. De hecho, la DMed, promoviendo un alto consumo de pescados y mariscos, se caracteriza por una adecuada proporción de ácidos grasos omega-6/ omega-3, favoreciendo un perfil menos inflamatorio que otras dietas occidentales, donde una alta ingesta de ácidos grasos omega- 6 determina mayor producción de citoquinas proinflamatorias $\mathrm{y}$ factores procoagulantes que aumentan el riesgo de enfermedades crónicas como diabetes mellitus $\mathrm{y}$ ateroesclerosis ${ }^{70}$.

\section{Probióticos}

Los probióticos son microorganismos vivos que, consumidos en cantidades adecuadas, confieren beneficios para la salud ${ }^{71-73}$. Los alimentos fermentados derivados de la leche, como yogurt y quesos, son parte de la DMed, y su ingesta reduciría marcadores inflamatorios asociados con el desarrollo de ateroesclerosis ${ }^{74}$. Sin embargo, el papel beneficioso de los microorganismos vivos lácteos no está totalmente comprobado ${ }^{75}$. Otros alimentos, como olivas, alcaparras, vinagre y vino, propios de esta dieta, también aportan probióticos que podrían contribuir a la microbiota intestinal $^{76-82}$.

\section{La alimentación tradicional chilena, una dieta de estilo mediterráneo}

Además de la cuenca del mar Mediterráneo, existen otros cuatro ecosistemas de tipo mediterráneo localizados entre los paralelos $30^{\circ}$ y $45^{\circ}$ de latitud norte o sur con sus costas mirando al occidente, entre los que se encuentra la región central de Chile ${ }^{83,84}$ (Figura 1). Asimismo, la producción agrícola de la zona central de nuestro país comprende productos similares a aquellos de 


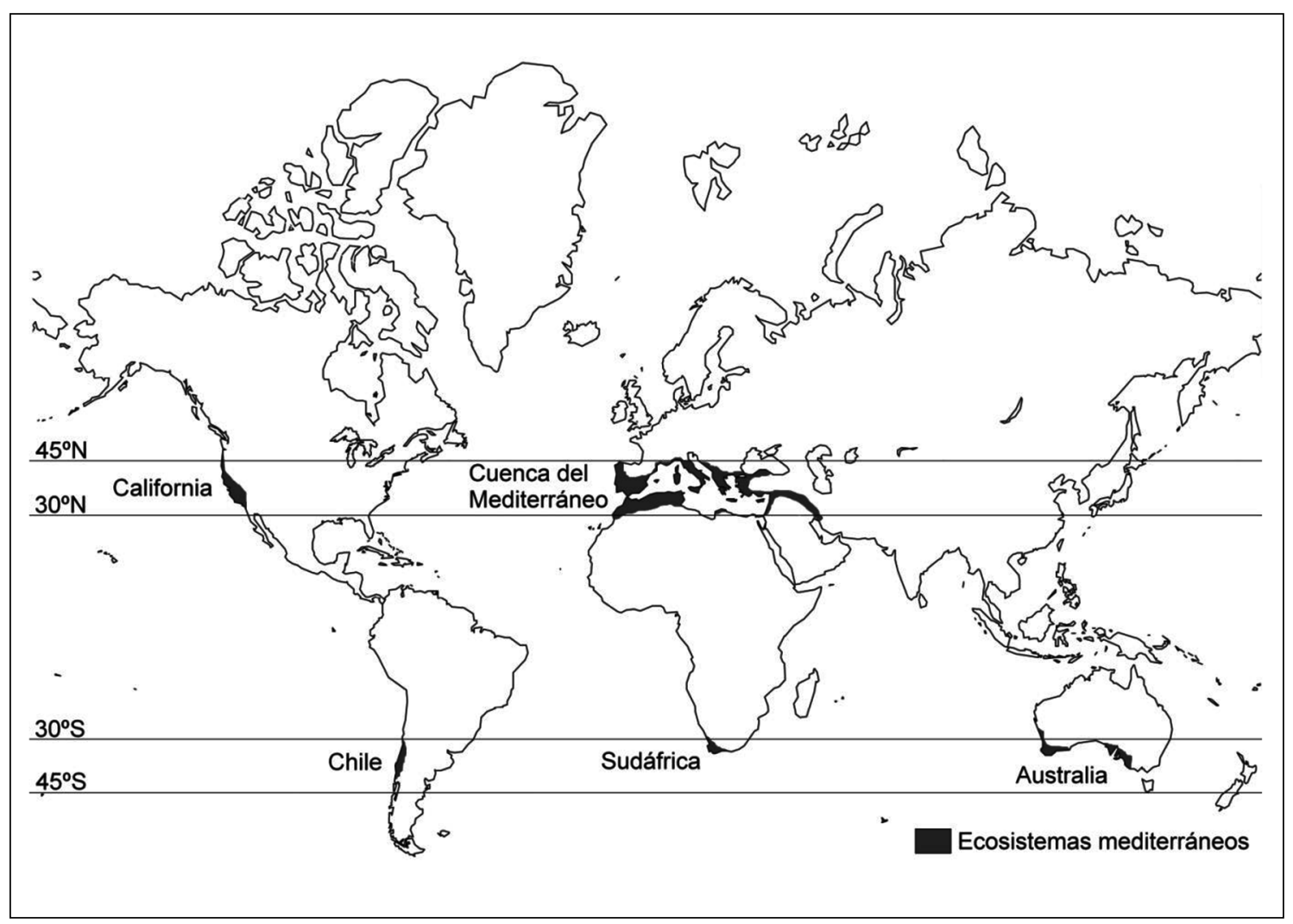

Figura 1. Ecosistemas mediterráneos en el mundo. Según la definición climática de Köppen ${ }^{55}$, existen cinco ecosistemas mediterráneos principales localizados entre las latitudes $30^{\circ}$ y $45^{\circ}$ de ambos hemisferios como se señala en el mapa.

países mediterráneos ${ }^{85}$, siendo el aceite de oliva y el vino los más representativos ${ }^{86}$.

Por otro lado, la gastronomía tradicional chilena posee varios platos distintivos que utilizan ingredientes y formas de preparación similares a la DMed. Así, platos como el charquicán y los porotos granados contienen productos vegetales cocidos y se preparan en base a un sofrito. Asimismo, la ensalada tomate con cebolla y los pebres son también mezclas ricas en antioxidantes y fibra, derivadas del uso de cebolla, ajo, tomate, perejil, ají y cilantro. Por otro lado, la palta es un alimento propio de nuestra cultura que se caracteriza por un bajo aporte de grasas saturadas y elevado en ácidos grasos monoinsaturados y fitoesteroles.

Utilizando los datos de disponibilidad de alimentos de la Organización de las Naciones Unidas para la Alimentación y la Agricultura (FAO), la dieta consumida por los chilenos en la década de
1990-99 mostraba similitudes a la DMed tradicional de España e Italia en $1960^{87}$. Sin embargo, existe la interrogante de si la dieta actual de los chilenos es efectivamente una dieta saludable de tipo mediterráneo. Para cuantificar la adherencia a la DMed en nuestra población, se ha desarrollado y aplicado un índice de alimentación mediterráneo ${ }^{88,89}$. Este índice fue diseñado homologando el patrón de alimentación mediterránea de Europa con los hábitos de consumo de alimentos similares o equivalentes en nuestra población y fue aplicado en un grupo de adultos mayores de Providencia ${ }^{90} \mathrm{y}$ de trabajadores de una empresa metal-mecánica ${ }^{89}$. En el grupo de trabajadores, una intervención en el casino con mediterranización de la dieta demostró un incremento significativo en este índice con importantes efectos en salud, incluyendo una disminución en la prevalencia de síndrome metabólico $^{89}$. Finalmente, el Programa Aliméntate Sano de nuestra universidad ha aplicado este 
índice de alimentación a través de una plataforma online (www.alimentatesano.cl) para público general desde 2010, lo que ha permitido hacer un seguimiento de este estilo de alimentación en Chile. Los datos muestran que solamente $10 \%$ de la población adulta chilena cumple con un patrón de alimentación de tipo mediterráneo (Figura 2).
En conjunto, estos estudios demuestran que el nivel de adherencia de nuestra población a este patrón dietario es bajo, lo cual podría mejorarse por medio de intervenciones dirigidas. La Tabla 3 presenta, en forma resumida, un conjunto de recomendaciones para aplicar una dieta de tipo mediterráneo en Chile.

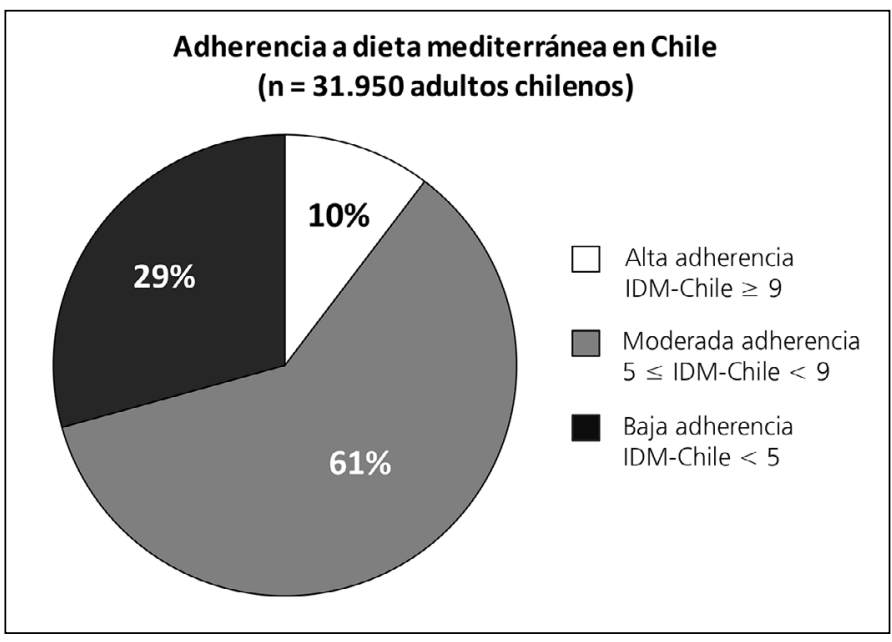

Figura 2. Calidad actual de la alimentación de los adultos chilenos en base a un patrón de consumo de tipo mediterráneo. Utilizando una muestra de 31.950 adultos chilenos, el Índice Chileno de Dieta Mediterránea (IDM-Chile) del Programa Aliméntate Sano ha permitido evaluar la adherencia de nuestra población a este tipo de dieta.

Tabla 3. Recomendaciones para la aplicación de una dieta mediterránea en Chile

1. Consuma verduras (lechuga, zanahoria, tomate, cebolla, espinaca, alcachofa, repollo, brócoli, apio, pimiento, zapallo italiano, berenjena, porotos verdes, pepino, etc.) crudas y cocidas en abundante cantidad, 3 o más porciones al día

2. Coma frutas, 2 o más unidades al día

3. Consuma cereales como pan, pastas, arroz a diario -de preferencia integrales- en moderada cantidad

4. Consuma productos lácteos, principalmente fermentados, yogurt y quesos, en moderada cantidad a diario. Si consume leche, debe ser descremada

5. Consuma legumbres (porotos, lentejas, garbanzos), al menos 3 veces por semana

6. Consuma pescados o mariscos frescos, congelados o en lata, al menos 3 veces por semana

7. Consuma carnes blancas como pavo, pollo o cerdo (nuevo cerdo), 2 a 4 veces por semana

8. Limite el consumo de carnes rojas (vaca y cordero), embutidos u otras carnes procesadas (salchichas, hamburguesas) a una vez a la semana

9. Coma huevos, 1 a 4 unidades por semana

10. Coma frutos secos como nueces y almendras, al menos dos veces por semana

11. Para cocinar y aliñar sus comidas use de preferencia aceite de oliva, si no, aceites vegetales como aceite Canola, rico en ácidos grasos monoinsaturados. El consumo de palta (de gran producción y consumo en Chile) en moderación, 1 a 3 unidades a la semana, también es una buena fuente de grasas saludables

12. Limite el consumo de crema, mantequilla y margarina; bebidas y alimentos azucarados; y papas fritas envasadas o similares a menos de 1 vez por semana

13. Use especias y condimentos (como limón, vinagre, ajo, menta, orégano, canela, etc.) para preparar sus comidas

14. Beba vino en moderada cantidad con las comidas ( 1 copa al día para mujeres y 2 copas al día para hombres) 


\section{Conclusiones e implicancias}

El consumo de un patrón de alimentos característicos de la DMed se asocia con numerosos beneficios para la salud. Estos beneficios se atribuyen a compuestos bioactivos que ejercen efectos sinérgicos y disminuyen el riesgo para el desarrollo de enfermedades crónicas.

La región central de Chile corresponde a uno de los ecosistemas mediterráneos del mundo cuya producción agropecuaria es rica en alimentos propios de la DMed, como el aceite de oliva y el vino tinto. Asimismo, numerosos platos típicos de la gastronomía chilena se preparan con alimentos característicos mediterráneos y usando técnicas culinarias similares a las usadas en esos países. Sin embargo, algunos estudios muestran una baja adherencia a este patrón dietario en segmentos de nuestra población, aunque carecemos de datos basados en una muestra con representativa nacional. Por lo tanto, Chile presenta grandes oportunidades y ofrece una enorme potencialidad para desarrollar e incrementar el consumo de alimentos característicos de una DMed.

Los esfuerzos de tipo poblacional global para controlar los estilos de alimentación no saludables en población adulta chilena han sido muy escasos y solamente en los últimos años se han intentado implementar algunas iniciativas. Algunos estudios recientes usando intervenciones basadas en el modelo de la DMed han mostrado beneficio en adultos $^{88}$. Mediante el diseño y la implementación de políticas públicas adecuadas, nuestro país podría acrecentar su producción de alimentos mediterráneos destinados al consumo local, contribuyendo a que la población chilena disponga de alimentos de mayor calidad nutricional que promuevan su salud previniendo la tasa creciente de enfermedades crónicas.

\section{Dedicatoria}

Este artículo está dedicado a la memoria del Dr. Federico Leighton Puga (1937-2012), médico investigador y pionero en el estudio y la promoción de la dieta mediterránea en Chile.

Agradecimientos: Los resultados de nuestro grupo mencionados en este artículo han sido obtenidos mediante proyectos financiados con fondos de FONDEF, Fundación Alimenta y Fundación Banmédica.

\section{Referencias}

1. WHO. Attaining the nine global noncommunicable diseases targets; a shared responsibility. Switzerland: 2014.

2. Simopoulos AP. Dietary omega-3 fatty acid deficiency and high fructose intake in the development of metabolic syndrome, brain metabolic abnormalities, and non-alcoholic fatty liver disease. Nutrients 2013; 5 (8): 2901-23.

3. de Lorgeril M, Salen P. Modified Cretan Mediterranean diet in the prevention of coronary heart disease and cancer. World Rev Nutr Diet 2000; 87: 1-23.

4. Chrysohoou C, Panagiotakos DB, Pitsavos C, Das UN, Stefanadis C. Adherence to the Mediterranean diet attenuates inflammation and coagulation process in healthy adults: The ATTICA Study. J Am Coll Cardiol 2004; 44 (1): 152-8.

5. Trichopoulou A. Traditional Mediterranean diet and longevity in the elderly: a review. Public Health Nutr 2004; 7 (7): 943-7.

6. Serra-Majem L, Roman B, Estruch R. Scientific evidence of interventions using the Mediterranean diet: a systematic review. Nutr Rev 2006; 64 (2 Pt 2): S27-47.

7. Estruch R, Ros E, Salas-Salvado J, Covas MI, Corella D, Aros F, et al. Primary prevention of cardiovascular disease with a Mediterranean diet. N Engl J Med 2013; 368 (14): 1279-90.

8. Sofi F, Macchi C, Abbate R, Gensini GF, Casini A. Mediterranean diet and health. Biofactors 2013; 39 (4): 335-42.

9. Dussaillant C, Echeverría G, Urquiaga I, Velasco N, Rigotti A. Evidencia actual sobre los beneficios de la dieta mediterránea en salud. Rev Med Chile 2016; 144 (8): 1044-52.

10. Keys A. Coronary heart disease in seven countries. Circulation 1970; 41 (4 Suppl): 1-211.

11. Keys A. Mediterranean diet and public health: personal reflections. Am J Clin Nutr 1995; 61 (6 Suppl): 1321S$3 \mathrm{~S}$.

12. Simopoulos AP. The Mediterranean diets: What is so special about the diet of Greece? The scientific evidence. J Nutr 2001; 131 (11 Suppl): 3065S-73S.

13. Saura-Calixto F, Goni I. Definition of the Mediterranean diet based on bioactive compounds. Crit Rev Food Sci Nutr 2009; 49 (2): 145-52.

14. Espósito K, Marfella R, Ciotola M, Di Palo C, Giugliano F, Giugliano G, et al. Effect of a mediterranean-style 
diet on endothelial dysfunction and markers of vascular inflammation in the metabolic syndrome: a randomized trial. Jama 2004; 292 (12): 1440-6.

15. Bulló M, Casas R, Portillo MP, Basora J, Estruch R, García-Arellano A, et al. Dietary glycemic index/load and peripheral adipokines and inflammatory markers in elderly subjects at high cardiovascular risk. Nutr Metab Cardiovasc Dis 2013; 23 (5): 443-50.

16. Konstantinidou V, Covas MI, Sola R, Fitó M. Up-to date knowledge on the in vivo transcriptomic effect of the Mediterranean diet in humans. Mol Nutr Food Res 2013; 57 (5): 772-83.

17. Schwingshackl L, Hoffmann G. Mediterranean dietary pattern, inflammation and endothelial function: a systematic review and meta-analysis of intervention trials. Nutrition, metabolism, and cardiovascular diseases: Nutr Metab Cardiovasc Dis 2014; 24 (9): 929-39.

18. García-Arellano A, Ramallal R, Ruiz-Canela M, Salas-Salvado J, Corella D, Shivappa N, et al. Dietary Inflammatory Index and Incidence of Cardiovascular Disease in the PREDIMED Study. Nutrients 2015; 7 (6): 4124-38.

19. Damasceno NR, Sala-Vila A, Cofan M, Pérez-Heras AM, Fito M, Ruiz-Gutiérrez V, et al. Mediterranean diet supplemented with nuts reduces waist circumference and shifts lipoprotein subfractions to a less atherogenic pattern in subjects at high cardiovascular risk. Atherosclerosis 2013; 230 (2): 347-53.

20. Bartolomé B, Monagas M, Garrido I, Gómez-Cordoves C, Martin-Álvarez PJ, Lebron-Aguilar R, et al. Almond (Prunus dulcis (Mill.) D.A. Webb) polyphenols: from chemical characterization to targeted analysis of phenolic metabolites in humans. Arch Biochem Biophys 2010; 501 (1): 124-33.

21. Estruch R, Martínez-González MA, Corella D, Salas-Salvado J, Ruiz-Gutiérrez V, Covas MI, et al. Effects of a Mediterranean-style diet on cardiovascular risk factors: a randomized trial. Ann Intern Med 2006; 145 (1): 1-11.

22. Fitó M, Guxens M, Corella D, Sáez G, Estruch R, de la Torre R, et al. Effect of a traditional Mediterranean diet on lipoprotein oxidation: a randomized controlled trial. Ann Intern Med 2007; 167 (11): 1195-203.

23. Mena MP, Sacanella E, Vazquez-Agell M, Morales M, Fito M, Escoda R, et al. Inhibition of circulating immune cell activation: a molecular antiinflammatory effect of the Mediterranean diet. Am J Clin Nutr 2009; 89 (1): 248-56.

24. Ros E, Martínez-González MA, Estruch R, Salas-Salvado J, Fitó M, Martínez JA, et al. Mediterranean diet and cardiovascular health: Teachings of the PREDIMED study. Adv Nutr 2014; 5 (3): 330S-6S.
25. Sesso HD, Liu S, Gaziano JM, Buring JE. Dietary lycopene, tomato-based food products and cardiovascular disease in women. J Nutr 2003; 133 (7): 2336-41.

26. Vilahur G, Cubedo J, Padro T, Casani L, Mendieta G, González A, et al. Intake of cooked tomato sauce preserves coronary endothelial function and improves apolipoprotein A-I and apolipoprotein J protein profile in high-density lipoproteins. Transl Res 2015; 166 (1): 44-56.

27. Talvas J, Caris-Veyrat C, Guy L, Rambeau M, Lyan B, Minet-Quinard R, et al. Differential effects of lycopene consumed in tomato paste and lycopene in the form of a purified extract on target genes of cancer prostatic cells. Am J Clin Nutr 2010; 91 (6): 1716-24.

28. Chen J, Song Y, Zhang L. Effect of lycopene supplementation on oxidative stress: an exploratory systematic review and meta-analysis of randomized controlled trials. Journal Med Food 2013; 16 (5): 361-74.

29. Grosso G, Buscemi S, Galvano F, Mistretta A, Marventano S, La Vela V, et al. Mediterranean diet and cancer: epidemiological evidence and mechanism of selected aspects. BMC Surg 2013; 13 Suppl 2: S14.

30. Ostan R, Lanzarini C, Pini E, Scurti M, Vianello D, Bertarelli C, et al. Inflammaging and cancer: a challenge for the Mediterranean diet. Nutrients 2015; 7 (4): 2589-621.

31. Devaraj S, Mathur S, Basu A, Aung HH, Vasu VT, Meyers S, et al. A dose-response study on the effects of purified lycopene supplementation on biomarkers of oxidative stress. J Am Coll Nutr 2008; 27 (2): 267 73.

32. Burton-Freeman B, Sesso HD. Whole food versus supplement: comparing the clinical evidence of tomato intake and lycopene supplementation on cardiovascular risk factors. Adv Nutr 2014; 5 (5): 457-85.

33. Martínez-González MA, Corella D, Salas-Salvado J, Ros E, Covas MI, Fiol M, et al. Cohort profile: design and methods of the PREDIMED study. Int J Epidemiol 2012; 41 (2): 377-85.

34. Vallverdu-Queralt A, de Alvarenga JF, Estruch R, Lamuela-Raventos RM. Bioactive compounds present in the Mediterranean sofrito. Food Chemistry 2013; 141 (4): 3365-72.

35. Halliwell B. Antioxidants and human disease: a general introduction. Nutr Reviews 1997; 55 (1 Pt 2): S44-9; discussion S9-52.

36. Halliwell B. Biochemistry of oxidative stress. Biochem Soc T 2007; 35 (Pt 5): 1147-50.

37. Sies H. Oxidative stress: a concept in redox biology and medicine. Redox Biology 2015; 4: 180-3.

38. Urquiaga I, Strobel P, Pérez D, Martínez C, Cuevas 
A, Castillo O, et al. Mediterranean diet and red wine protect against oxidative damage in young volunteers. Atherosclerosis 2010; 211 (2): 694-9.

39. Tresserra-Rimbau A, Rimm EB, Medina-Remon A, Martínez-González MA, de la Torre R, Corella D, et al. Inverse association between habitual polyphenol intake and incidence of cardiovascular events in the PREDIMED study. Nutrition, metabolism, and cardiovascular diseases: NMCD 2014; 24 (6): 639-47.

40. Williams RJ, Spencer JP. Flavonoids, cognition, and dementia: actions, mechanisms, and potential therapeutic utility for Alzheimer disease. Free Radic Biol Med 2012; 52 (1): 35-45.

41. Galleano M, Verstraeten SV, Oteiza PI, Fraga CG. Antioxidant actions of flavonoids: thermodynamic and kinetic analysis. Arch Biochemistry Biophys 2010; 501 (1): 23-30.

42. Middleton E, Jr., Kandaswami C, Theoharides TC. The effects of plant flavonoids on mammalian cells: implications for inflammation, heart disease, and cancer. Pharmac Reviews 2000; 52 (4): 673-751.

43. Bonaccio M, Pounis G, Cerletti C, Donati MB, Iacoviello L, de Gaetano G. Mediterranean diet, dietary polyphenols and low-grade inflammation: results from the moli-sani study. British J Clin Pharmacol 2017; 83: 107-13.

44. Schewe T, Kuhn H, Sies H. Flavonoids of cocoa inhibit recombinant human 5-lipoxygenase. J Nutr 2002; 132 (7): 1825-9.

45. Leopold JA, Loscalzo J. Oxidative risk for atherothrombotic cardiovascular disease. Free Radic Biol Med 2009; 47 (12): 1673-706.

46. Vauzour D, Rodríguez-Mateos A, Corona G, Oruna-Concha MJ, Spencer JP. Polyphenols and human health: prevention of disease and mechanisms of action. Nutrients 2010; 2 (11): 1106-31.

47. Chatzianagnostou K, Del Turco S, Pingitore A, Sabatino L, Vassalle C. The Mediterranean Lifestyle as a Non-Pharmacological and Natural Antioxidant for Healthy Aging. Antioxidants 2015; 4 (4): 719-36.

48. Grassi D, Desideri G, Ferri C. Flavonoids: antioxidants against atherosclerosis. Nutrients 2010; 2 (8): 889-902.

49. Cuevas AM, Guasch V, Castillo O, Irribarra V, Mizon C, San Martin A, et al. A high-fat diet induces and red wine counteracts endothelial dysfunction in human volunteers. Lipids 2000; 35 (2): 143-8.

50. Sies H. Polyphenols and health: update and perspectives. Arch Biochem Biophys 2010; 501 (1): 2-5.

51. Leikert JF, Rathel TR, Wohlfart P, Cheynier V, Vollmar AM, Dirsch VM. Red wine polyphenols enhance endothelial nitric oxide synthase expression and subsequent nitric oxide release from endothelial cells. Circulation 2002; 106 (13): 1614-7.

52. Hugel HM, Jackson N, May B, Zhang AL, Xue CC. Polyphenol protection and treatment of hypertension. Phytomedicine 2016; 23 (2): 220-31.

53. Sies H, Stahl W, Sevanian A. Nutritional, dietary and postprandial oxidative stress. J Nutr 2005; 135 (5): 96972.

54. Urquiaga I, Guasch V, Marshall G, San Martin A, Castillo O, Rozowski J, et al. Effect of Mediterranean and Occidental diets, and red wine, on plasma fatty acids in humans. An intervention study. Biological Research 2004; 37 (2): 253-61.

55. Natella F, Macone A, Ramberti A, Forte M, Mattivi F, Matarese RM, et al. Red wine prevents the postprandial increase in plasma cholesterol oxidation products: a pilot study. Brit J Nutr 2011; 105 (12): 1718-23.

56. Gorelik S, Ligumsky M, Kohen R, Kanner J. A novel function of red wine polyphenols in humans: prevention of absorption of cytotoxic lipid peroxidation products. FASEB J 2008; 22 (1): 41-6.

57. Kanner J, Gorelik S, Roman S, Kohen R. Protection by polyphenols of postprandial human plasma and low-density lipoprotein modification: the stomach as a bioreactor. Journal Agr Food Chem 2012; 60 (36): 87906.

58. Davis CD, Milner JA. Gastrointestinal microflora, food components and colon cancer prevention. J Nutr Biochem 2009; 20 (10): 743-52.

59. Threapleton DE, Greenwood DC, Evans CE, Cleghorn CL, Nykjaer C, Woodhead C, et al. Dietary fibre intake and risk of cardiovascular disease: systematic review and meta-analysis. BMJ 2013; 347: f6879.

60. Evans CE, Greenwood DC, Threapleton DE, Cleghorn CL, Nykjaer C, Woodhead CE, et al. Effects of dietary fibre type on blood pressure: a systematic review and meta-analysis of randomized controlled trials of healthy individuals. J Hypertens 2015; 33 (5): 897-911.

61. Wu Y, Qian Y, Pan Y, Li P, Yang J, Ye X, et al. Association between dietary fiber intake and risk of coronary heart disease: A meta-analysis. Clin Nutr 2015; 34 (4): 603-11.

62. Howarth NC, Saltzman E, Roberts SB. Dietary fiber and weight regulation. Nutr Rev 2001; 59 (5): 129-39.

63. Slavin JL. Dietary fiber and body weight. Nutrition 2005; 21 (3): 411-8.

64. Klingberg S, Ellegard L, Johansson I, Hallmans G, Weinehall L, Andersson $\mathrm{H}$, et al. Inverse relation between dietary intake of naturally occurring plant sterols and serum cholesterol in northern Sweden. Am J Clin Nutr 2008; 87 (4): 993-1001. 
65. Escurriol V, Cofan M, Serra M, Bullo M, Basora J, Salas-Salvado J, et al. Serum sterol responses to increasing plant sterol intake from natural foods in the Mediterranean diet. Eur J Nutr 2009; 48 (6): 373-82.

66. Covas MI, de la Torre R, Fito M. Virgin olive oil: a key food for cardiovascular risk protection. Br J Nutr 2015; 113 Suppl 2: S19-28.

67. Hohmann CD, Cramer H, Michalsen A, Kessler C, Steckhan N, Choi K, et al. Effects of high phenolic olive oil on cardiovascular risk factors: A systematic review and meta-analysis. Phytomedicine 2015; 22 (6): 631-40.

68. Vitaglione P, Savarese M, Paduano A, Scalfi L, Fogliano V, Sacchi R. Healthy virgin olive oil: a matter of bitterness. Crit Rev Food Sci 2015; 55 (13): 1808-18.

69. Simopoulos AP. Evolutionary aspects of diet: the omega-6/omega-3 ratio and the brain. Mol Neurobiol 2011; 44 (2): 203-15.

70. Simopoulos AP. The importance of the omega-6/omega-3 fatty acid ratio in cardiovascular disease and other chronic diseases. Exp Biol Med (Maywood) 2008; 233 (6): 674-88.

71. Parvez S, Malik KA, Ah Kang S, Kim HY. Probiotics and their fermented food products are beneficial for health. J App Microbiol 2006; 100 (6): 1171-85.

72. Guarner F, Requena T, Marcos A. Consensus statements from the Workshop "Probiotics and Health: Scientific evidence”. Nutr Hosp 2010; 25 (5): 700-4.

73. Fontana L, Bermúdez-Brito M, Plaza-Díaz J, Munoz-Quezada S, Gil A. Sources, isolation, characterisation and evaluation of probiotics. Br J Nutr 2013; 109 Suppl 2: S35-50.

74. Fernández M, Hudson JA, Korpela R, de los Reyes-Gavilán CG. Impact on human health of microorganisms present in fermented dairy products: an overview. BioMed research international 2015; 2015: 412714.

75. Tapsell LC. Fermented dairy food and CVD risk. Br J Nutr 2015; 113 Suppl 2: S131-5.

76. Kabak B, Dobson AD. An introduction to the traditional fermented foods and beverages of Turkey. Crit Rev Food Science Nutrition 2011; 51 (3): 248-60.

77. Queipo-Ortuno MI, Boto-Ordonez M, Murri M, Gómez-Zumaquero JM, Clemente-Postigo M, Estruch R, et al. Influence of red wine polyphenols and ethanol on the gut microbiota ecology and biochemical biomarkers. Am J Clin Nutr 2012; 95 (6): 1323-34.

78. Clemente-Postigo M, Queipo-Ortuno MI, Boto-Ordonez M, Coin-Araguez L, Roca-Rodríguez MM, Del-
gado-Lista J, et al. Effect of acute and chronic red wine consumption on lipopolysaccharide concentrations. Am J Clin Nutr 2013; 97 (5): 1053-61.

79. Hurtado A, Reguant C, Bordons A, Rozes N. Lactic acid bacteria from fermented table olives. Food Microbiology 2012; 31 (1): 1-8.

80. Vitali B, Minervini G, Rizzello CG, Spisni E, Maccaferri S, Brigidi P, et al. Novel probiotic candidates for humans isolated from raw fruits and vegetables. Food Microbiology 2012; 31 (1): 116-25.

81. Argyri AA, Zoumpopoulou G, Karatzas KA, Tsakalidou E, Nychas GJ, Panagou EZ, et al. Selection of potential probiotic lactic acid bacteria from fermented olives by in vitro tests. Food Microbiology 2013; 33 (2): 282-91.

82. Nychas GJ, Panagou EZ, Parker ML, Waldron KW, Tassou CC. Microbial colonization of naturally black olives during fermentation and associated biochemical activities in the cover brine. Lett Applied Microbiol 2002; 34 (3): 173-7.

83. Köppen V. Das geographische System der Klimate. Köppen V, Geiger R, editors. Berlin: Gebrüder Borntraeger; 1936.

84. Groves RH. Biogeography of Mediterranean invasions. Groves RH, di Castri F, editors. Cambridge: Cambridge University Press; 1992.

85. Diamond J. Guns, Germs and Steel: The Fates of Human Societies. Diamond J, editor. New York: Norton; 1997.

86. Urquiaga I, Echeverría G, Polic G, Castillo O, Liberona Y, Rozowski J, et al. Mediterranean food and diets, global resource for the control of metabolic syndrome and chronic diseases. World Rev Nutr Diet 2008; 98: 150-73.

87. Rozowski J, Castillo O. Is the Chilean diet a Mediterranean-type diet? Biol Res 2004; 37 (2): 313-9.

88. Echeverría G, Urquiaga I, Concha MJ, Dussaillant C, Villarroel L, Velasco N, Leighton F, Rigotti A. Validación de cuestionario autoaplicable para un índice de alimentación mediterránea en Chile. Rev Med Chile 2016; 144: 1531-43.

89. Leighton F, Polic G, Strobel P, Pérez D, Martínez C, Vásquez L, et al. Health impact of Mediterranean diets in food at work. Public Health Nutrition 2009; 12 (9A): 1635-43.

90. Castillo O, Rozowski J, Cuevas A, Maiz A, Soto M, Mardones $\mathrm{F}$, et al. [Nutrients intake in elderly people living in Providencia, Santiago de Chile]. Rev Med Chile 2002; 130 (12): 1335-42. 